\title{
Association of SLC1A2 and SLC17A7 polymorphisms with major depressive disorder in a Thai population
}

\author{
Benjamard Thaweethee ${ }^{1,2}$, Sirijit Suttajit ${ }^{3}$, Samur Thanoi ${ }^{1,2}$, Caroline F. Dalton ${ }^{4}$, Gavin P. Reynolds ${ }^{2,4}$, \\ Sutisa Nudmamud-Thanoi ${ }^{1,2, *}$
}

Abstract

Background: Major depressive disorder (MDD) is a common psychiatric disorder with high prevalence and high risk of suicide. Genetic variation of glutamate transporters may associate with MDD and suicide attempt.

Objectives: To evaluate polymorphisms of excitatory amino acid transporter 2 gene (SLC1A2; rs752949, rs1885343, rs4755404, and rs4354668) and vesicular glutamate transporter 1 gene (SLC17A7; rs1043558, rs2946848, and rs11669017) in patients with MDD with and without suicide attempt, and determine the association of these polymorphisms with age of onset and severity of MDD.

Methods: DNA was extracted from blood taken from patients with MDD $(\mathrm{n}=100$; including nonsuicidal [ $\mathrm{n}=50$ ] and suicidal $[\mathrm{n}=50]$ subgroups) and controls $(\mathrm{n}=100)$. Genotyping was conducted using TaqMan single-nucleotide polymorphism (SNP) genotyping.

Results: We found a significant difference in $S L C 17 A 7$ rs2946848 genotype distribution between patients in the MDD and control groups $(P=0.016)$. Moreover, significant differences in SLC1A2 rs752949 $(P=0.022)$ and SLC17A7 rs2946848 $(P=0.026)$ genotype distributions were observed between patients in the nonsuicidal MDD and suicidal MDD groups. SLC1A2 rs1885343 A allele carriers showed significantly lower age of onset than GG genotype $(P=0.049)$. Furthermore, the severity of MDD indicated by the Hamilton Depression Rating Scale (HDRS) score of G allele carriers of SLC1A2 rs4755404 was significantly greater than the CC genotype $(P=0.013)$.

Conclusions: Polymorphisms of SLC1A2 and SLC17A7 may contribute to the risk of MDD and/or suicide attempt. An association of an $S L C 1 A 2$ polymorphism with the severity of MDD was apparent.

Keywords: excitatory amino acid transporter 2, gene; major depressive disorder; single-nucleotide polymorphisms; SLC1A2; SLC17A7; suicide, attempted; vesicular glutamate transporter 1

Major depressive disorder (MDD) or major depression is a common psychiatric disorder in the general population [1]. According to the World Health Organization (WHO), MDD affects more than 350 million people worldwide. Moreover, the Burden of Disease (BOD) Research Program in 2013 revealed that a Thai population of around 1.5 million people

*Correspondence to: Sutisa Nudmamud-Thanoi, Department of Anatomy, Faculty of Medical Science, Naresuan University, Phitsanulok 65000, Thailand, e-mail: sutisat@nu.ac.th

'Department of Anatomy, Faculty of Medical Science, Naresuan University, Phitsanulok 65000, Thailand

${ }^{2}$ Centre of Excellence in Medical Biotechnology, Faculty of Medical Science, Naresuan University, Phitsanulok 65000, Thailand

${ }^{3}$ Department of Psychiatry, Faculty of Medicine, Chiang Mai University, Chiang Mai 50200, Thailand

${ }^{4}$ Biomolecular Sciences Research Centre, Sheffield Hallam University, Sheffield S1 1WB, UK

O Open Access. ( 2018 B. Thaweethee et al., published by Sciendo. (cc)BY-NC-ND This work is licensed under the Creative Commons Attribution NonCommercial-NoDerivatives 4.0 License. 
suffered from depression [2]. Psychiatric disorders, especially MDD, have been strongly linked to suicide and suicide attempts [3-6]. A metaanalysis has found that approximately $90 \%$ of the people who commit suicide have been diagnosed with a psychiatric disorder before death [7]. Moreover, the risk of suicide in depressed women and girls is 20 -fold that of the general population [8]. MDD is thus considered a severe psychiatric disorder.

Both attempted suicide and completed suicide are highly familial [9], in which completed suicide shows a higher concordance in monozygotic twins (11.3\%) than in dizygotic twins (1.8\%) [10]. These findings indicate that genetic factors are involved in suicidal behavior. Genetic variation, especially single-nucleotide polymorphisms (SNPs), of the glutamatergic system has attracted attention in the study of psychiatric disorders. Glutamate, a major excitatory neurotransmitter in the central nervous system, plays important roles in balancing brain functions and modulating various mood states. The packaging and release of vesicular glutamate can be influenced by stress and psychotropic drugs [11]. Abnormalities of glutamate concentrations in brain areas that involve mood and emotion have been recognized in patients with MDD [12-14]. Increases in glutamate levels in plasma and serum have been found in patients with MDD [15-18], in whom these increases may be a result of abnormalities of glutamate transporters. A postmortem study has demonstrated a downregulation of expression of the gene for excitatory amino acid transporter 2 (EAAT2) $(S L C 1 A 2)$ in anterior cingulate cortex and dorsolateral prefrontal cortex of patients with MDD [19]. Moreover, a low EAAT2 protein expression was found in the orbitofrontal cortex of patients with MDD [20]. Furthermore, a decreased expression of the gene for vesicular glutamate transporter 1 (VGLUT1) (SLC17A7) was found in the entorhinal cortex of patients with either MDD or bipolar disorder [21], and in middle temporal gyrus in suicide victims of severe psychiatric disorders including MDD, bipolar disorder, and schizophrenia [21]. By contrast, increased $S L C 17 A 7$ expression was observed in the anterior cingulate cortex of patients with schizophrenia [22]. Therefore, genes encoding glutamate transporters may vary in psychiatric diseases including MDD and warrant genetic study.

To our knowledge, there are only a few studies reporting genetic polymorphisms of SLC1A2 and SLC1A7 in MDD. Most studies have reported an association of SNPs of SLC1A2 and $S L C 17 A 7$ with schizophrenia [23-28] and bipolar disorder $[29,30]$. A study of SLC1A2 rs4755404 has reported protective and risk genetic variants for suicide [31]. Genetic polymorphisms of SLC1A2 and SLC17A7 may confer vulnerability for MDD and suicidal behavior. Understanding the association between genetic polymorphisms and MDD including suicide may be valuable in improving its prevention, diagnosis, and effective treatment. Therefore, we aimed to determine the association of such gene polymorphisms with MDD and suicidal behavior or ideation. The association of gene polymorphisms of SLC1A2 and SLC17A7 with the age of onset and severity of MDD in MDD patients was investigated.

\section{Methods}

\section{Subjects}

All experimental protocols used in the present study were approved by the Human Ethics Committee of Naresuan University (certificate of approval No. 524/2017) and Faculty of Medicine, Chiang Mai University (certificate of approval No. 314/2017). All participants were fully informed about the study, and documented informed consent was obtained individually from each participant. The participants (18-65 years old) were divided into 2 groups: MDD and control ( $\mathrm{n}=100$ per group). MDD was diagnosed by a psychiatrist in the Department of Clinical Psychiatry, Maharaj Nakorn Chiang Mai Hospital, Thailand, based on criteria in the Diagnostic and Statistical Manual of Mental Disorders, fifth edition (DSM-5). The Hamilton Depression Rating Scale (Thai version; Thai HDRS-17) was used to indicate depression. Patients who had a history of schizophrenia, bipolar disorder, or drug addiction were excluded from the present study. The participants in the MDD group were divided into 2 subgroups ( $\mathrm{n}=50$ per subgroup) including patients with MDD without suicide attempt (nonsuicidal MDD group) and those with suicide attempt (suicidal MDD group) according to the Mini International Neuropsychiatric Interview (MINI; Thai version 5.0.0-revised 2007). The duration of illness, the period that patients suffered depressive symptoms before diagnosis of MDD, and age of onset were also recorded. The control group included healthy volunteer participants who had no previous history of schizophrenia, mood disorder, anxiety, or drug addiction. The age and sex of the control participants were matched with those of the patients with MDD. The mental health of the participants in the control group was evaluated using the Thai Mental Health Indicator (TMHI-66). Participants who identified with abnormal mental health were excluded from the study.

\section{Blood sample collection and DNA extraction}

Fingertip blood samples were dropped on Whatman Flinders Technology Associations (FTA) cards (GE Healthcare) and allowed to dry completely at room temperature. Dried blood samples were kept at room temperature following the manufacturer's instructions until used. The blood samples 
were collected over approximately 6 months. Following the instruction manual for the FTA cards, genomic DNA stored on FTA cards at room temperature for over 17 years has been successfully amplified by polymerase chain reaction (PCR). Dried blood spots were punched using a Harris Micro Punch $2.0 \mathrm{~mm}$ (GE Healthcare), and then DNA was extracted using Chelex 100 resin (Bio-Rad). Briefly, FTA disks were washed 3 times in $1 \mathrm{~mL}$ high purify water at room temperature for $10 \mathrm{~min}$ with occasional inversion. After discarding all liquid, FTA disks were incubated in $5 \%$ Chelex 100 resin at $56^{\circ} \mathrm{C}$ for $20 \mathrm{~min}$ and mixed by vortexing. FTA disks were further incubated at $100^{\circ} \mathrm{C}$ for $8 \mathrm{~min}$ and centrifuged at $20,000 \times g$ for $3 \mathrm{~min}$. Supernatant containing DNA was carefully collected into a new tube and kept at $-80^{\circ} \mathrm{C}$ until use. The DNA concentration was measured using a NanoDrop ND-1000 spectrophotometer (Thermo Scientific) according to the manufacturer's protocol.

\section{SNP study}

SNPs of SLC1A2 including rs752949, rs1885343, rs4755404, and rs4354668 and SNPs of SLC17A7 including rs1043558, rs2946848, and rs11669017 were selected following previous reports in association with psychiatric disorders. From LDlink calculation, rs752949 has high linkage disequilibrium with rs1885343 $\left(\mathrm{D}^{\prime}=0.93\right.$ in Chinese Dai in Xishuangbanna, China), in which a role for EAAT2 protein expression in the nucleus accumbens was reported [32]. Previous studies have reported an association of rs4755404 with schizophrenia [23] and suicide [31]. rs4354668 is supposed to play important roles in patients with schizophrenia [24] and bipolar disorder $[25,29]$. A trend towards the association of rs1043558 with schizophrenia has been found in psychiatric patients [28]. Although the functional roles of rs2946848 and rs11669017 have not been reported to our knowledge, rs 2946848 located at the promoter region and rs11669017 located near the $5^{\prime}$ position might be involved with transcription factor binding sites. SNP information was collected from the SNP database (dbSNP; https://www.ncbi.nlm.nih.gov/snp/) and the Ensembl project (http://www.ensembl.org/index.html).

Each SNP genotyping was examined separately using a TaqMan SNP genotyping real-time PCR. PCR reactions were conducted in a total volume of $10 \mu \mathrm{L}$ containing $10 \mathrm{ng}$ of DNA template, $1 \times$ TaqMan GTXpress Master Mix (Thermo Fisher Scientific), nuclease-free water, and $1 \times$ predesigned TaqMan SNP Genotyping Assays (Assay ID: C_ 605534_20 for rs752949, C__1292328_10 for rs1885343, C_27982507_10 for rs4755404, C_27142767_10 for rs4354668, C_ 9493385_10 for rs1043558, C_11494903_10 for rs2946848, and C_27843812_10 for rs11669017; Thermo
Fisher Scientific). PCR amplification was conducted for 40 cycles with PCR conditions comprising pre-PCR read stage at $60^{\circ} \mathrm{C}$ for $30 \mathrm{~s}$, DNA polymerase activation at $95^{\circ} \mathrm{C}$ for $10 \mathrm{~min}$, denaturation at $95^{\circ} \mathrm{C}$ for $15 \mathrm{~s}$, annealing/extension at $60^{\circ} \mathrm{C}$ for $1 \mathrm{~min}$, and post-PCR read stage at $60^{\circ} \mathrm{C}$ for $30 \mathrm{~s}$ with StepOnePlus and QuantStudio 3 Real-Time PCR Systems (Applied Biosystems). Subsequently, the genotypes were determined and clustered using StepOne Software or QuantStudio Software (Applied Biosystems).

\section{Statistical analysis}

The Statistical Package for the Social Sciences (SPSS Statistics for Windows; version 17.0) was used to perform a Pearson $\chi^{2}$ test to analyze differences in sex and genotype frequency between the 2 groups. Univariate analysis of variance and an independent samples $t$ test were used to analyze differences in age, age of onset, duration of illness, and HDRS score frequency between the 2 groups. A Hardy-Weinberg equilibrium test was conducted using PLINK software (version 1.07). Differences with $P<0.05$ in tests of statistical inference were considered significant.

\section{Results}

The demographic data of participants showed no significant difference of age and sex between those in the MDD and control groups $(P>0.05)$. The duration of illness in patients in the suicidal MDD group was significantly longer than that in patients in the nonsuicidal MDD group $(P=0.024)$, while age, sex, age of onset, and HDRS scores were not significantly different between the groups ( $P>0.05$; Table 1). Female patients with MDD had significantly higher HDRS scores than male patients with $\operatorname{MDD}(P=0.004$; Table 1).

All SNPs of SLC1A2 and SLC17A7 in the control group were in Hardy-Weinberg equilibrium $(P>0.05$; data not shown). The significant values of genotype distribution in the comparison of common homozygous to minor allele carriers (dominant model) between patients in the MDD group and volunteers in the control groups, and between patients in the nonsuicidal MDD and suicidal MDD groups were stronger than the 3 genotype comparisons (additive model), and these results are then used in further analysis. The distribution of genotype frequencies of SLC17A7 rs2946848 was significantly different between patients in the MDD group and volunteers in the control group $(P=0.016)$, in which the $\mathrm{C}$ allele carrier (CC/CT genotype) was more common in patients in the MDD group (Table 2). 
Table 1. Demographic data and clinical characteristics of the participants in the present study

\begin{tabular}{|c|c|c|c|c|c|c|}
\hline Variable (mean \pm SD) or $n(\%)$ & $\begin{array}{l}\text { Control } \\
(n=100)\end{array}$ & $\begin{array}{c}\text { MDD } \\
(n=100)\end{array}$ & $P$ & $\begin{array}{l}\text { Nonsuicidal MDD } \\
\quad(n=50)\end{array}$ & $\begin{array}{l}\text { Suicidal MDD } \\
\quad(n=50)\end{array}$ & $\boldsymbol{P}$ \\
\hline Age (years), & $44.31 \pm 14.77$ & $43.74 \pm 15.00$ & $0.79^{+}$ & $44.38 \pm 15.32$ & $44.24 \pm 14.35$ & $0.96^{+}$ \\
\hline \multicolumn{7}{|l|}{ Sex } \\
\hline Female & $74(74)$ & $75(75)$ & $0.94^{+}$ & $39(78)$ & $35(70)$ & $0.36^{+}$ \\
\hline Male & $26(26)$ & $25(25)$ & & $11(22)$ & $15(30)$ & \\
\hline Age of onset (years old) & & & & $38.34 \pm 16.28$ & $33.66 \pm 13.93$ & $0.12^{+}$ \\
\hline Duration of illness (years) & & & & $6.05 \pm 8.44$ & $10.58 \pm 11.18$ & $0.024^{\dagger *}$ \\
\hline HDRS-17 (scores) & & & & $11.66 \pm 7.35$ & $13.48 \pm 7.44$ & $0.22^{+}$ \\
\hline Female & & $13.82 \pm 7.75$ & $0.004^{\ddagger *}$ & $12.33 \pm 7.76$ & $15.49 \pm 7.50$ & $0.08^{+}$ \\
\hline Male & & $9.00 \pm 4.93$ & & $9.27 \pm 5.26$ & $8.80 \pm 4.86$ & $0.47^{\dagger}$ \\
\hline
\end{tabular}

${ }^{\dagger} M D D$ group vs. control group or nonsuicidal MDD group vs. suicidal MDD group

${ }^{\ddagger}$ Female MDD patients vs. male MDD patients

${ }^{*} P<0.05$

HDRS, Hamilton Depression Rating Scale; MDD, major depressive disorder

Moreover, the distribution of genotype frequencies of SLC1A2 rs752949 and SLC17A7 rs2946848 was significantly different between patients in the nonsuicidal MDD and suicidal MDD groups ( $P=0.022$ and $P=0.026$, respectively) in which the T allele carrier (TT/CT genotype) of rs752949 and TT genotype of SLC17A7 rs2946848 were more common in patients in the suicidal MDD group. We did not find any significantly different genetic distributions of SLC1A2 rs1885343, SLC1A2 rs4755404, SLC1A2 rs4354668, SLC17A7 rs1043558, and SLC17A7 rs11669017 between patients in the MDD group and volunteers in the control group, or between patients in the nonsuicidal MDD and suicidal MDD groups (Table 2).

The association study of gene polymorphisms with age of onset and HDRS scores in patients with MDD demonstrated that A allele carriers (AA/AG genotype) of SLC1A2 rs1885343 had a significantly lower age of onset than patients with MDD who were GG homozygotes $(P=0.049$; Figure 1A) (33.80 \pm 14.36 and $40.09 \pm 16.23$, respectively). Moreover, the HDRS scores of patients with MDD who were $G$ allele carriers (GG/CG genotype) of SLC1A2 rs4755404 were significantly lower than those of patients with MDD and a common CC genotype $(P=0.013$; Figure 1B; $11.37 \pm 6.94$ and $15.37 \pm 7.84$, respectively). We found no significant association of SLC1A2 rs752949, SLC1A2 rs4354668, SLC17A7 rs1043558, SLC17A7 rs2946848, and SLC17A7 rs11669017 with either age of onset or HDRS scores. Nor did we find any significant association of the duration of illness with SNPs.

\section{Discussion}

In the present study, we found an association of $S L C 17 A 7$ rs2946848 with MDD. We showed that C allele carriers (CC/ CT) of SLC17A7 rs2946848 were more common among MDD patients. According to the MatInspector software analysis, the SNP rs2946848 is in intron 2 and is predicted to locate in the promoter region of $S L C 17 A 7$. This SNP might interrupt mRNA transcription of $S L C 17 A 7$ leading to a glutamate transporter abnormality in those with MDD. Our findings are consistent with those of other studies that have found differential changes in SLC17A7 expression in bipolar disorder [21] and schizophrenia [22].

The function of SNPs of glutamate transporter-related genes in those with MDD is unclear. The present study found an association of SLC1A2 rs752949 and SLC17A7 rs2946848 with suicide attempt in patients with MDD. Bearing a T allele (TT/CT) of SLC1A2 rs752949 and TT genotype of SLC17A7 rs2946848 may be risk factors for suicide attempt in those with MDD. To our knowledge, the associations of these 2 SNPs with MDD or other psychiatric disorders have not been reported previously. Here we report evidence of an association of SLC1A2 rs752949 and SLC17A7 rs2946848 with MDD.

We found that the duration of illness in patients with MDD and suicidal attempt is longer than that in patients with MDD who did not attempt suicide (nonsuicidal MDD group). Our findings are consistent with a previous study that found a correlation between suicidal symptoms and the duration of illness 
Table 2. Genotype frequencies of SLC1A2 and SLC17A7 polymorphisms in the control and MDD groups

\begin{tabular}{|c|c|c|c|c|c|c|}
\hline \multirow[b]{2}{*}{ SLC1A2 rs752949 } & \multirow[t]{2}{*}{$\mathbf{n}$} & \multicolumn{3}{|c|}{ Genotype (frequency) } & \multirow[t]{2}{*}{${ }^{\dagger} \boldsymbol{P}$} & \multirow[t]{2}{*}{${ }^{\ddagger} \boldsymbol{P}$} \\
\hline & & CC & $\mathbf{C T}$ & TT & & \\
\hline Control & 100 & $74(0.74)$ & $26(0.26)$ & $0(0.00)$ & 0.25 & 0.23 \\
\hline MDD & 100 & $81(0.81)$ & $18(0.18)$ & $1(0.01)$ & & \\
\hline Nonsuicidal & 50 & $45(0.90)$ & $4(0.08)$ & $1(0.02)$ & $0.023^{*}$ & $0.022^{*}$ \\
\hline Suicidal & 50 & $36(0.72)$ & $14(0.28)$ & $0(0.00)$ & & \\
\hline SLC1A2 rs 1885343 & & AA & AG & GG & & \\
\hline Control & 100 & $17(0.17)$ & $51(0.51)$ & $32(0.32)$ & 0.16 & 0.65 \\
\hline MDD & 100 & $26(0.26)$ & $39(0.39)$ & $35(0.35)$ & & \\
\hline Nonsuicidal & 50 & $14(0.28)$ & $17(0.34)$ & $19(0.38)$ & 0.59 & 0.53 \\
\hline Suicidal & 50 & $12(0.24)$ & $22(0.44)$ & $16(0.32)$ & & \\
\hline SLC1A2 rs4755404 & & CC & CG & GG & & \\
\hline Control & 100 & $33(0.33)$ & $45(0.45)$ & $22(0.22)$ & 0.89 & 0.65 \\
\hline MDD & 100 & $30(0.30)$ & $46(0.46)$ & $24(0.24)$ & & \\
\hline Nonsuicidal & 50 & $12(0.24)$ & $27(0.54)$ & $11(0.22)$ & 0.25 & 0.19 \\
\hline Suicidal & 50 & $18(0.36)$ & $19(0.38)$ & $13(0.26)$ & & \\
\hline SLC1A2 rs4354668 & & GG & GT & TT & & \\
\hline Control & 100 & $46(0.46)$ & $47(0.47)$ & $7(0.07)$ & 0.52 & 0.32 \\
\hline MDD & 100 & $53(0.53)$ & $39(0.39)$ & $8(0.08)$ & & \\
\hline Nonsuicidal & 50 & $27(0.54)$ & $21(0.42)$ & $2(0.04)$ & 0.39 & 0.84 \\
\hline Suicidal & 50 & $26(0.52)$ & $18(0.36)$ & $6(0.12)$ & & \\
\hline SLC17A7 rs1043558 & & GG & GT & TT & & \\
\hline Control & 100 & $25(0.25)$ & $55(0.55)$ & $20(0.20)$ & 0.85 & 0.74 \\
\hline MDD & 100 & $23(0.23)$ & $59(0.59)$ & $18(0.18)$ & & \\
\hline Nonsuicidal & 50 & $9(0.18)$ & $32(0.64)$ & $9(0.18)$ & 0.47 & 0.24 \\
\hline Suicidal & 50 & $14(0.28)$ & $27(0.54)$ & $9(0.18)$ & & \\
\hline SLC17A7 rs2946848 & & CC & CT & TT & & \\
\hline Control & 100 & $4(0.04)$ & $36(0.36)$ & $60(0.60)$ & $0.047^{*}$ & $0.016^{*}$ \\
\hline MDD & 100 & $8(0.08)$ & $49(0.49)$ & $43(0.43)$ & & \\
\hline Nonsuicidal & 50 & $3(0.06)$ & $31(0.62)$ & $16(0.32)$ & $0.034^{*}$ & $0.026^{*}$ \\
\hline Suicidal & 50 & $5(0.10)$ & $18(0.36)$ & $27(0.54)$ & & \\
\hline SLC17A7 rs11669017 & & CC & CG & GG & & \\
\hline Control & 100 & $24(0.24)$ & 49 (0.49) & $27(0.27)$ & 0.69 & 0.87 \\
\hline MDD & 100 & $19(0.19)$ & $53(0.53)$ & $28(0.28)$ & & \\
\hline Nonsuicidal & 50 & $14(0.28)$ & $23(0.46)$ & $13(0.26)$ & 0.07 & 0.66 \\
\hline Suicidal & 50 & $5(0.10)$ & $30(0.60)$ & $15(0.30)$ & & \\
\hline
\end{tabular}

${ }^{\dagger}$ MDD group vs. control group or nonsuicidal-MDD group vs. suicidal-MDD group

${ }^{\ddagger}$ Analysis with common homozygotes vs. minor allele carriers

${ }^{*} P<0.05$

MDD, major depressive disorder 

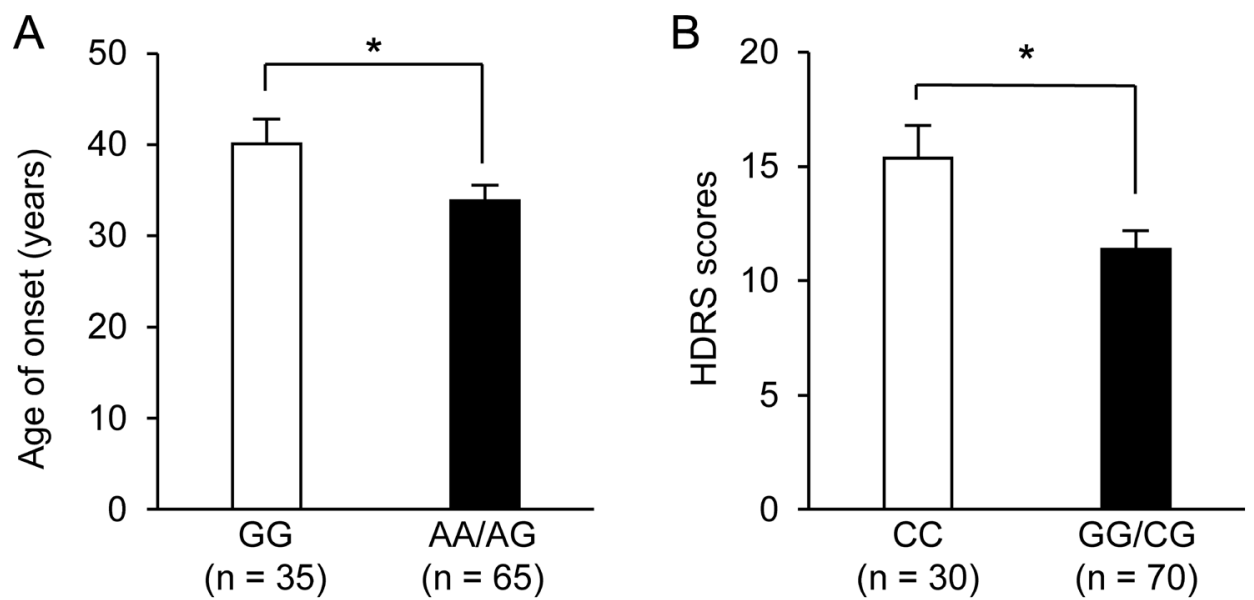

Figure 1. Association of gene polymorphisms with age of onset and HDRS scores in MDD patients. (A) Age of onset (years) of common GG homozygotes and A allele carriers (AA/AG genotype) of SLC1A2 rs1885343 and (B) HDRS scores of common CC homozygotes and G allele carriers (GG/CG genotype) of SLC1A2 rs4755404 in MDD patients $(n=100)$. Bars with black or white fill represent mean data, error bars SEM. ${ }^{*} P<0.05$; independent samples $t$ test.

HDRS, Hamilton Depression Rating Scale; MDD, major depressive disorder; SEM, standard error of the mean

in patients with MDD [33]. Degeneration of brain, in which decreased hippocampal volume is correlated with the duration of depression has been reported for patients with MDD [34-36]. Moreover, patients with MDD who have attempted suicide were found to have smaller hippocampal volume than those patients with MDD who had not attempted suicide [37]. These findings suggest that the depression might somehow progressively reduce hippocampal volume with consequent abnormalities in brain function, which may be reflected by psychiatric symptoms.

We found an association of SLC1A2 rs1885343 with the age of MDD onset in patients we studied. Those with a GG genotype of SLC1A2 rs1885343 showed a significantly greater age of MDD onset than those patients who were A allele carriers (AA/AG genotype). SNP rs1885343 is located at intron 1 of SLC1A2, where it may have a functional effect on EAAT2 expression. Supporting this effect of SLC1A2 rs1885343, our previous study reported an influence of rs1885343 on EAAT2 expression in the nucleus accumbens of healthy volunteers in whom those with the GG genotype showed lower expression of EAAT2 than those who were A allele carriers (AA/ AG) [32]. Therefore, SLC1A2 rs1885343 might contribute to changes of EAAT2 expression in people with MDD, as consistent with previous reports of reduced cortical expression of SLC1A2 and EAAT2 in those with MDD [19, 20].

As consistent with a previous report [8], we found that symptom severity in patients with MDD is positively correlated with sex in women and girls who have greater risk than men and boys. Moreover, the present study found that SLC1A2 rs4755404 was associated with symptom severity in patients with MDD. The CC genotype of this SNP showed higher HDRS scores. The CC genotype may also influence gene expression because in patients with schizophrenia it is associated with a significant increase in the EAAT2 exon 9 skipping splice variant mRNA expression in the anterior cingulate cortex pyramidal cells compared with that in those with a G genotype [26].

This preliminary study is limited by its small sample size. Moreover, although a Bonferroni correction has been made for multiple testing, only SLC17A7 rs2946848 showed a significant association with MDD. Therefore, a further study in a substantially larger sample size determined by a power analysis is needed to confirm the gene polymorphism associations with MDD and suicide.

\section{Conclusions}

This study provides initial evidence for SLC1A2 and SLC17A7 polymorphisms as risk factors for MDD and/or suicide attempt. Moreover, an association of SLC1A2 polymorphism with the severity of MDD is suggested. These possible risk factors may be predictive markers for diagnosis and helpful in development of an effective treatment.

Author contributions. BT, SS, ST, CFD, GPR, and SN-T contributed substantially to the conception and design of this study. BT and SS contributed substantially to the acquisition of data. BT, ST, CFD, GPR, and SN-T analyzed and interpreted the data. BT, SS, ST, and CFD drafted the manuscript. GPR and SN-T contributed substantially to its critical revision. All the authors approved the final version submitted for 
publication and take responsibility for the statements made in the published article.

Acknowledgments. We are very grateful to all the participants, to the staff of Clinical Psychiatry, Maharaj Nakorn Chiang Mai Hospital, Thailand, and the graduate students of Department of Anatomy, Faculty of Medical Science, Naresuan University, Thailand, for data and blood collection and the Biomolecular Sciences Research Centre, Sheffield Hallam University, UK, for support. This work was supported by the Naresuan University Research Fund, funding from the Commission of Higher Education, the Thailand Research Fund through the Royal Golden Jubilee Ph.D. Program (Grant No. PHD/0297/2552) and the British Council Newton Fund, and supported in part by Chiang Mai University. The present work was presented in part as a poster at the 41st Annual Conference of the Anatomy Association of Thailand (AAT41), 2018 May 23-25, Cha-am, Phetchaburi, Thailand. Benjamard Thaweethee, Sirijit Suttajit, Samur Thanoi, Caroline F. Dalton, Gavin P. Reynolds, Sutisa Nudmamud-Thanoi. Association of SLC1A2 and SLC17A7 gene polymorphisms with major depressive disorder in a Thai population [PP 05, AAT157].

Conflict of interest statement. The authors have each completed and submitted an International Committee of Medical Journal Editors Uniform Disclosure Form for Potential Conflicts of Interest. None of the authors have any conflict of interest to disclose.

\section{References}

[1] Joyce PR. Epidemiology of mood disorders. In: Gelder M, Andreasen N, Lopez-Ibor J, Geddes J, editors. New Oxford Textbook of Psychiatry. 3rd ed. Oxford: Oxford University Press; 2012, 645-50.

[2] Kittirattanapaiboon P, Tantirangsee N, Chutha W, Tanaree A, Kwansanit P, Assanangkornchai S, Thai Mental Health Survey Working Group. Prevalence of mental disorders and mental health problems: Results from Thai national mental health survey 2013. Nonthaburi: Department of Mental Health, Ministry of Public Health. [online]. 2016 [cited 2017 Jan 15]; Available from: https:// www.dmh.go.th/ebook/view.asp?id=382

[3] Rich CL, Young D, Fowler RC. San Diego suicide study. I. Young vs old subjects. Arch Gen Psychiatry. 1986; 43:577-82.

[4] Henriksson MM, Aro HM, Marttunen MJ, Heikkinen ME, Isometsa ET, Kuoppasalmi KI, et al. Mental disorders and comorbidity in suicide. Am J Psychiatry. 1993; 150:935-40.

[5] Conwell Y, Duberstein PR, Cox C, Herrmann JH, Forbes NT, Caine ED. Relationships of age and axis I diagnoses in victims of completed suicide: a psychological autopsy study. Am J Psychiatry. 1996; 153:1001-8.

[6] Harwood D, Hawton K, Hope T, Jacoby R. Psychiatric disorder and personality factors associated with suicide in older people: a descriptive and case-control study. Int J Geriatr Psychiatry. 2001; 16:155-65.

[7] Arsenault-Lapierre G, Kim C, Turecki G. Psychiatric diagnoses in 3275 suicides: a meta-analysis. BMC psychiatry. 2004; 4:1-37.

[8] Chesney E, Goodwin GM, Fazel S. Risks of all-cause and suicide mortality in mental disorders: a meta-review. World Psychiatry. 2014; 13:153-60.

[9] Brent DA, Mann JJ. Family genetic studies, suicide, and suicidal behavior. Am J Med Genet C Semin Med Genet. 2005; 133c:13-24.

[10] Roy A, Segal NL, Centerwall BS, Robinette CD. Suicide in twins. Arch Gen Psychiatry. 1991; 48:29-32.

[11] Musazzi L, Milanese M, Farisello P, Zappettini S, Tardito D, Barbiero VS, et al. Acute stress increases depolarization-evoked glutamate release in the rat prefrontal/frontal cortex: the dampening action of antidepressants. PloS One. 2010; 5:e8566. doi: 10.1371/journal. pone.0008566

[12] Hashimoto K, Sawa A, Iyo M. Increased levels of glutamate in brains from patients with mood disorders. Biol Psychiatry. 2007; 62:1310-6.

[13] Mirza Y, Tang J, Russell A, Banerjee SP, Bhandari R, Ivey J, et al. Reduced anterior cingulate cortex glutamatergic concentrations in childhood major depression. J Am Acad Child Adolesc Psychiatry. 2004; 43:341-8.

[14] Rosenberg DR, Macmaster FP, Mirza Y, Smith JM, Easter PC, Banerjee SP, et al. Reduced anterior cingulate glutamate in pediatric major depression: a magnetic resonance spectroscopy study. Biol Psychiatry. 2005; 58:700-4.

[15] Altamura CA, Mauri MC, Ferrara A, Moro AR, D’Andrea G, Zamberlan F. Plasma and platelet excitatory amino acids in psychiatric disorders. Am J Psychiatry. 1993; 150:1731-3.

[16] Kim JS, Schmid-Burgk W, Claus D, Kornhuber HH. Increased serum glutamate in depressed patients. Arch Psychiatr Nervenkr (1970). 1982; 232:299-304.

[17] Mauri MC, Ferrara A, Boscati L, Bravin S, Zamberlan F, Alecci M, Invernizzi G. Plasma and platelet amino acid concentrations in patients affected by major depression and under fluvoxamine treatment. Neuropsychobiology. 1998; 37:124-9.

[18] Mitani H, Shirayama Y, Yamada T, Maeda K, Ashby CR, Jr, Kawahara R. Correlation between plasma levels of glutamate, alanine and serine with severity of depression. Prog Neuropsychopharmacol Biol Psychiatry. 2006; 30:1155-8.

[19] Choudary PV, Molnar M, Evans SJ, Tomita H, Li JZ, Vawter MP, et al. Altered cortical glutamatergic and GABAergic signal transmission with glial involvement in depression. Proc Natl Acad Sci U S A. 2005; 102:15653-8.

[20] Miguel-Hidalgo JJ, Waltzer R, Whittom AA, Austin MC, Rajkowska G, Stockmeier CA. Glial and glutamatergic markers in depression, alcoholism, and their comorbidity. J Affect Disord. 2010; 127:230-40.

[21] Uezato A, Meador-Woodruff JH, McCullumsmith RE. Vesicular glutamate transporter mRNA expression in the medial temporal lobe in major depressive disorder, bipolar disorder, and schizophrenia. Bipolar Disord. 2009; 11:711-25.

[22] Oni-Orisan A, Kristiansen LV, Haroutunian V, Meador-Woodruff $\mathrm{JH}, \mathrm{McCullumsmith} \mathrm{RE}$. Altered vesicular glutamate transporter expression in the anterior cingulate cortex in schizophrenia. Biol Psychiatry. 2008; 63:766-75.

[23] Deng X, Shibata H, Ninomiya H, Tashiro N, Iwata N, Ozaki N, Fukumaki Y. Association study of polymorphisms in the excitatory amino acid transporter 2 gene (SLC1A2) with schizophrenia. BMC Psychiatry. 2004; 4:21. doi: 10.1186/1471-244X-4-21 
[24] Spangaro M, Bosia M, Zanoletti A, Bechi M, Cocchi F, Pirovano A, et al. Cognitive dysfunction and glutamate reuptake: effect of EAAT2 polymorphism in schizophrenia. Neurosci Lett. 2012; 522:151-5.

[25] Poletti S, Radaelli D, Bosia M, Buonocore M, Pirovano A, Lorenzi C, et al. Effect of glutamate transporter EAAT2 gene variants and gray matter deficits on working memory in schizophrenia. Eur Psychiatry. 2014; 29:219-25.

[26] O’Donovan SM, Hasselfeld K, Bauer D, Simmons M, Roussos P, Haroutunian V, et al. Glutamate transporter splice variant expression in an enriched pyramidal cell population in schizophrenia. Transl Psychiatry. 2015; 5:1-9. doi: 10.1038/tp.2015.74

[27] Zhang B, Guan F, Chen G, Lin H, Zhang T, Feng J, et al. Common variants in SLC1A2 and schizophrenia: Association and cognitive function in patients with schizophrenia and healthy individuals. Schizophr Res. 2015; 169:128-34.

[28] Shen Y-C, Liao D-L, Chen J-Y, Wang Y-C, Lai I-C, Liou Y-J, et al. Resequencing and association study of vesicular glutamate transporter 1 gene (VGLUT1) with schizophrenia. Schizophr Res. 2009; 115:254-60.

[29] Dallaspezia S, Poletti S, Lorenzi C, Pirovano A, Colombo C, Benedetti F. Influence of an interaction between lithium salts and a functional polymorphism in SLC1A2 on the history of illness in bipolar disorder. Mol Diagn Ther. 2012; 16:303-9.

[30] Poletti S, Locatelli C, Radaelli D, Lorenzi C, Smeraldi E, Colombo C, Benedetti F. Effect of early stress on hippocampal gray matter is influenced by a functional polymorphism in EAAT2 in bipolar disorder. Prog Neuropsychopharmacol Biol Psychiatry. 2014; 51:146-52.

[31] Murphy TM, Ryan M, Foster T, Kelly C, McClelland R, O'Grady $\mathrm{J}$, et al. Risk and protective genetic variants in suicidal behaviour: association with SLC1A2, SLC1A3, 5-HTR1B \&NTRK2 polymorphisms. Behav Brain Funct. 2011; 7:1-9.

[32] Kerdsan W, Thanoi S, Nudmamud-Thanoi S, Reynolds GP. An association between genotypic variations and protein expression of the glial glutamate transporter 2 in the human nucleus accumbens. Neurosci Lett. 2012; 523:108-10.

[33] Javed MA. Suicidal symptoms in depressed Pakistani patients. J Pak Med Assoc. 1996; 46:69-70.

[34] Sheline YI, Gado MH, Kraemer HC. Untreated depression and hippocampal volume loss. Am J Psychiatry. 2003; 160:1516-8.

[35] Sheline YI, Sanghavi M, Mintun MA, Gado MH. Depression duration but not age predicts hippocampal volume loss in medically healthy women with recurrent major depression. J Neurosci. 1999; 19:5034-43.

[36] Frodl T, Jäger M, Smajstrlova I, Born C, Bottlender R, Palladino $\mathrm{T}$, et al. Effect of hippocampal and amygdala volumes on clinical outcomes in major depression: a 3-year prospective magnetic resonance imaging study. J Psychiatry Neurosci. 2008; 33:423-30.

[37] Colle R, Chupin M, Cury C, Vandendrie C, Gressier F, Hardy P, et al. Depressed suicide attempters have smaller hippocampus than depressed patients without suicide attempts. J Psychiatr Res. 2015; 61:13-8. 